\title{
CAMBIOS EN LA VEGETACIÓN DURANTE EL HOLOCENO RECIENTE EN EL VALLE DEL LOZOYA (SIERRA DE GUADARRAMA, MADRID)
}

Trabajo presentado a las

XXII Jornadas de la Sociedad Española de Paleontología.

Universidad de León

León, 28-30 de Septiembre de 2006

\author{
M. Blanca RUIZ ZAPATA ${ }^{1}$, Clemencia GÓMEZ \\ GONZÁLEZ1, José Antonio LÓPEZ SÁEZ², María \\ José GIL GARCÍA ${ }^{1}$, María Soledad VERA ${ }^{3}$, Rosa \\ MEDIAVILLA4, Fernando DOMÍNGUEZ ${ }^{4}$ y Juan \\ SANTISTEBAN ${ }^{5}$
}

\footnotetext{
${ }^{1}$ Departamento de Geología. Edificio de Ciencias. Universidad de Alcalá. 28871 Alcalá de Henares. blanca.ruiz@uah.es

${ }^{2}$ Laboratorio de Arqueobotánica. Departamento de Prehistoria. Instituto de Historia. CSIC. Duque de Medinaceli, 6. 28014 Madrid. alopez@ih.csic.es

${ }^{3}$ Departamento de Química Analítica. Edificio de Ciencias. Universidad de Alcalá. 28871 Alcalá de Henares. soledad,vera@uah.es

${ }^{4}$ Instituto Geológico y Minero de España. Ríos Rosas, 23. 28003 Madrid. r.mediavilla@igme.es

${ }^{5}$ Departamento de Estratigrafía. Universidad Complutense de Madrid. 28040 Madrid juancho@geo.ucm.es
}

Ruiz Zapata, B., Gómez González, C., López Sáez, J. A., Gil García, M. J., Vera, M. S., Mediavilla, R., Domínguez, F. \& Santisteban, J. 2007. Cambios en la vegetación durante el Holoceno reciente en el valle del Lozoya (Sierra de Guadarrama, Madrid). [Vegetation change during the Late Holocene in the Lozoya valley (Guadarrama Range, Madrid).] Revista Española de Paleontología, 22 (1), 95-102. ISSN 0213-6937.

\section{ABSTRACT}

Here we present palynological analyses carried avow a peaty located near to Rascafría village (Lozoya valley

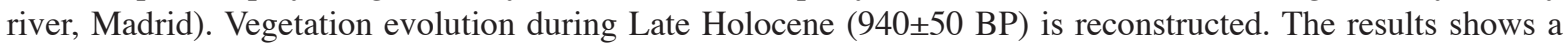
landscape dominated by herbaceous taxa, constituted mainly Cichorioideae, Asteraceae, Chenopodiaceae, Poaceae and Plantago lanceolata. Shrubs such as Erica arborea, Rosaceae, Juniperus and Cistaceae have certain role in the vegetation. Woodlands with Pinus sylvestris, P. pinaster and Quercus, Corylus and Betula are also noticeable throushout the sequence. Non-pollen palynomorphs like Pseudoschizaea circula and Glomus are used to infer the importance of erosive processes in situ. This information shows an open mediterranean landscape, due mainly to human activities, and changes in peatbog trophic condition. Changes in values of Alnus, Fraxinus and Cyperaceae are used to detected humid changes in the area. The information provided by $\mathrm{pH}(5,4-6,2)$ and sediment conductivity $(10-160 \mu \mathrm{S} / \mathrm{cm})$ serves to assess vegetation and land uses changes.

Key Words: palaeoecology, historical biogeography, palynology, Late Holocene, vegetation change.

\section{RESUMEN}

Se presentan los datos palinológicos procedentes de un testigo obtenido en una formación higroturbosa de origen periglacial situada en el término municipal de Rascafría (Valle del Lozoya, Madrid). El objetivo principal de este trabajo es reconstruir la evolución de la vegetación en el área durante el Holoceno Reciente. Los resultados obtenidos reflejan la existencia de un paisaje vegetal dominado por el estrato herbáceo, constituido fundamentalmente por Cichorioideae, Asteraceae, Chenopodiaceae, Poaceae y Plantago lanceolata. El grupo arbustivo (Erica arborea, Rosaceae, Juniperus y Cistaceae) tiene un papel poco importante en la vegetación; la masa forestal de Pinus sylvestris y en menor proporción P. pinaster y Quercus, junto a presencias de Betula, y Corylus, es poco representativa a lo largo del perfil, excepto hacia el techo de la secuencia, donde muestra un fuerte y progresivo aumento. De los microfósiles no polínicos destacamos la presencia de Pseudoschizaea circula y Glomus, que puede relacionarse con los procesos erosivos in situ favorecidos por fenómenos de so- 
brepastoreo. Esta información pone de manifiesto la presencia de un paisaje mediterráneo abierto y antropizado, dominado por zonas de pastizal dedicadas al ganado, según se deduce en el diagrama polínico a través de las proporciones de los componentes nitrófilos de carácter zoógeno y antropozoógeno. La presencia de taxones de ribera (Alnus y Fraxinus) y las variaciones de Cyperaceae, como representante mayoritario de los taxones higrohidrófilos, definen importantes fluctuaciones en la tasa de humedad. La información proporcionada por los valores del $\mathrm{pH}(5,4-6,2)$ y de la conductividad $(10-160 \mu \mathrm{S} / \mathrm{cm})$ del sedimento, constatan los cambios detectados en la vegetación y en los usos del suelo.

\section{Palabras clave: paleoecología, biogeografía, palinología, Holoceno Reciente, Cambios en la vegetación.}

\section{INTRODUCCIÓN}

A partir de los datos procedentes del análisis polínico llevado a cabo en una turbera en Rascafría (Madrid) se pretende reconstruir la evolución de la vegetación en el área durante el Holoceno Reciente. La turbera RAS, se localiza en el término municipal de Rascafría entre la carretera M-604 y la urbanización los Grifos (coordenadas UTM X: 427300, Y: 4529336) a una altitud de 1180 msnm (Fig. 1). En este entorno afloran arcosas y sedimentos silíceos paleocenos sobre los que se depositaron limos, arenas y conglomerados cuaternarios. Desde el punto de vista de la vegetación y de acuerdo a las características de zonación biogeográfica (Peinado Lorca \& Rivas-Martínez, 1987), la zona de estudio se encuentra enmarcada en el reino Holártico, distrito Guadarramense y piso bioclimático Supramediterráneo medio. En el mapa de series de vegetación del Valle del Paular (Fernández, 1988), el área de estudio se localiza dentro de la serie Luzulo forsteri-Querceto pyrenaicae correspondiente a bosques climatófilos de roble melojo (Quercus pyrenaica) que se desarrollan sobre suelos silíceos. La vegetación del entorno está constituida por formaciones dominadas por árboles y arbustos planocaducifolios, entre los que se encuentran abedules, fresnos,

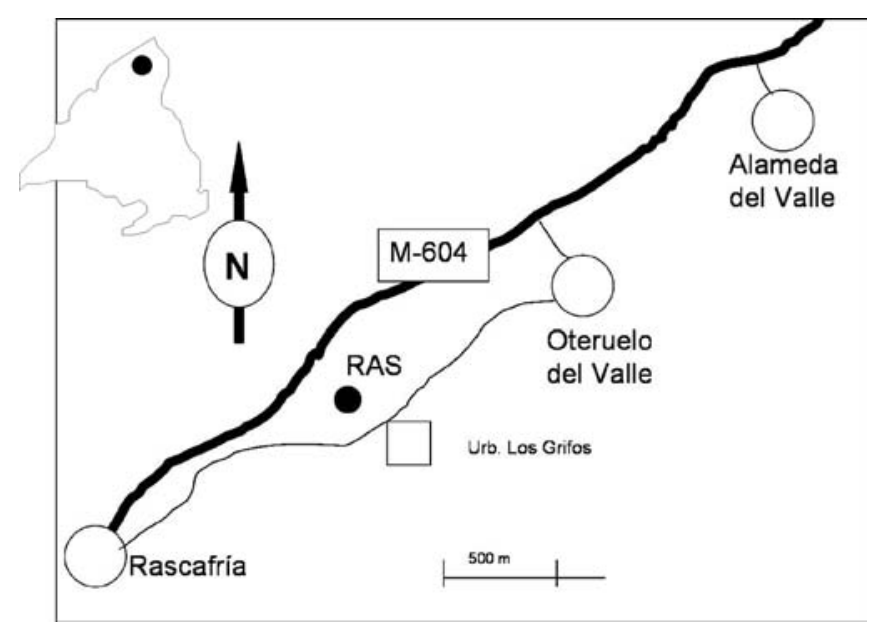

Figura 1. Situación geográfica de la turbera RAS (Rascafría, Madrid).

Geographical location of RAS peatbog (Rascafría, Madrid). melojos, sauces, avellanos, madreselvas, entre otros; en las laderas de la sierra y hacia el fondo del valle, se extienden melojares y pinares de P. sylvestris (Franco, 1995) y en el propio valle, donde se localiza la turbera objeto de este trabajo, se desarrollan los pastizales dedicados al ganado. La temperatura media anual está entre $8^{\circ}$ y $13^{\circ} \mathrm{C}$, la media de las mínimas del mes mas frío entre $-4^{\circ}$ y $-1^{\circ}$ $\mathrm{C}$, y la media de las máximas del mes mas frío entre $2^{\circ}$ y $9^{\circ} \mathrm{C}$. El índice de termicidad está entre 60 y 120 (Peinado Lorca \& Rivas-Martínez, 1987).

\section{MATERIAL Y MÉTODOS}

El testigo analizado (RAS) se obtuvo con una sonda manual tipo Rusa, alcanzando una profundidad de $60 \mathrm{~cm}$. Para la extracción de los granos de polen se utilizó la metodología clásica (Faegri et al., 1989) y el residuo fue sometido a técnicas de enriquecimiento, mediante la concentración del polen por flotación en licor de Thoulet (densidad 2).

La lectura y recuento del contenido polínico se llevo a cabo de acuerdo al método propuesto por Cambón (1981). Para la determinación de los tipos polínicos se tomó como referencia la palinoteca de la Universidad de Alcalá y se realizó de acuerdo a Valdés et al. (1987), Moore et al. (1991) y Reille (1992). La diferenciación de Pinus pinaster se basó en Arobba (1979), Díaz-Laviada (1989) y Mateus (1992).

Los datos de polen se presentan como frecuencia relativa de polen de cada taxón en un diagrama polínico elaborado mediante los programas informáticos TILIA-TILIA GRAPH y TGView 1.6.2 (Grimm, 1987). Para facilitar la lectura del diagrama se han señalado con presencias los taxones con valores menores al $1 \%$ y para los que su valor oscila entre el $1 \%$ y el $5 \%$, se ha utilizado una exageración del $5 \%$.

Los taxones se han agrupado de acuerdo a su procedencia arbórea, arbustiva y herbácea y su ordenación en cada grupo responde a la similitud de las exigencias ecológicas, al conocimiento de la vegetación del entorno estudiado y a su lluvia polínica actual (en realización por los autores), con objeto de facilitar la interpretación de los datos; así se han utilizado términos como: bosque ripario (Alnus, Fraxinus y Corylus); taxones nitrófilos antrópicos (Cichorioideae, Papaveraceae, Boraginaceae, Rumex y Artemisia); taxones nitrófilos antrópozoógenos (Urtica dioica tipo, Plantago lanceolata, Rubiaceae, Chenopodiaceae/Amaranthaceae), pastizales vivaces de origen antropozoógeno o pastos de siega, procedentes general- 
mente de pastizales anuales por pastoreo: Poaceae, Fabaceae, Caryophyllaceae, Brassicaceae, y taxones ubiquistas (Saxifraga, Sanguisorba, Solanaceae, Valerianaceae, entre otros). Adicionalmente los tipos esporales y microfósiles no polínicos también se han agrupado de acuerdo con sus afinidades ecológicas en coprófilos: 55A (van Geel, 1978, 1986; van Geel et al., 1981, 1983, 2003; López Sáez et al., 2000; López Sáez \& López García, 2003; Marinova \& Atanassova, 2006; Prager et al., 2006), 113 (Ahmed \& Cain, 1972; van Geel, 2001; van Geel et al., 2003; van Geel \& Aptroot, 2006; Blackford \& Innes, 2006), 165 (van Geel et al., 1983; Carrión, 2001) y 368 (van Geel et al., 1981, 1983, 2003; Cugny, 2004; Almeida-Lenero et al., 2005; van Geel \& Aptroot, 2006; Marinova \& Atanassova, 2006); tipos Eutróficos: 140 (van der Wiel, 1983; van Geel et al., 1983, 2003; van Geel \& Aptroot, 2006; Marinova \& Atanassova, 2006), 181 (van Geel et al., 1983), 182 (van Geel et al., 1983), 332D (van Geel et al., 1981; van Geel et al., 1989; Riera et al., 2006), 353A (van Geel et al., 1981; Haas, 1996) y 731 (Bakker \& van Smeerdijk, 1982), y tipo Oligotrófico: 170 (Pals et al., 1980; van Geel et al., 1983, 1989; López Sáez et al., 2005).

Las muestras de suelo, fueron secadas al aire y tamizadas. Las medidas de $\mathrm{pH}$ y conductividad se llevaron a cabo de acuerdo con la norma ISO 10390:2005; así se prepararon suspensiones de suelo en agua (1:5) agitando vigorosamente durante 10 minutos. A continuación se mantuvieron en reposo para llevar a cabo las medidas de $\mathrm{pH}$ y conductividad en el sobrenadante, utilizando un $\mathrm{pH}$-metro Inolab y un conductímetro Crison. En todo momento, la temperatura de trabajo ha sido $25 \pm 1{ }^{\circ} \mathrm{C}$.

Se han realizado tres dataciones (Tabla 1), dos de ellas en Beta Analytic Inc. y la tercera en Gliwice Radiocarbon Laboratory. Los datos han sido calibrados usando OxCal version 3.10 (Bronk-Ramsey, 1995, 2001).

\section{RESULTADOS}

En el análisis polínico de la secuencia RAS, se han identificado un total de 43 taxones de los cuales 10 son arbóreos, 4 arbustivos y 23 herbáceos, junto a 6 taxones acuáticos, además de 25 microfósiles no polínicos y esporas.

Los hechos más destacados en el diagrama polínico RAS (Fig. 2) se resumen en el dominio de polen herbáceo determinado por la abundancia de los taxones nitrófilos antrópicos (principalmente de Cichorioideae, Asteraceae y Papaveraceae), de pastizales vivaces de origen antropozoógeno (Poaceae, Fabaceae y Brassicaceae) y de los taxones nitrófilos antropozoógenos (Plantago lanceolata, Urtica dioica y Chenopodiaceae/Amaranthaceae).

El componente arbustivo está formado básicamente por Ericaceae, Rosaceae, Juniperus y Cistaceae; en cuanto a la masa forestal, en donde están representados los elementos de carácter regional (Gil, 1992; Franco, 1995; Franco et al., 1998; Uzquiano, 2002), está constituida fundamentalmente por Pinus y Quercus, se encuentra también presente en el diagrama polínico Betula; además de taxones de ribera (Alnus, Fraxinus y Corylus). Los taxones higro-hidrófilos (Fig. 3) están representados por Cyperaceae, Ranunculaceae, Nymphaceae, Polygonum y Typha. Dentro de los palinomorfos no polínicos destacan Pseudoschizaea circula y los tipos 140, 170, 181, 207, 351, 368 y 731 .

En cuanto a la dinámica de la vegetación, la relación de los taxones arbóreos/arbustivos/herbáceos, la sustitución entre los componentes de los elementos arbustivos/herbáceos y relación polen/microfósiles no polínicos/condiciones ecológicas permiten establecer tres zonas polínicas:

ZONA I: abarca la parte inferior del diagrama polínico (31-60 cm). El tramo de turba correspondiente a los 31$33 \mathrm{~cm}$ de profundidad, comprendida entre $2455 \pm 50 \mathrm{BP}$ y $920 \pm 50$ BP. Desde el punto de vista litológico corresponde de base a techo con limos arenosos. En cuanto a la vegetación, Cichorioideae, Asteraceae, Poaceae y Plantago lancelolata son los taxones más abundantes. Se observa el aumento de Plantago lanceolata coincidente con la aparente disminución de Cichorioideae, Poaceae y Papaveraceae. El componente arbustivo esta representado por Rosaceae, Juniperus y Erica arborea, con valores inferiores al 5\%. El estrato arbóreo está poco representado siendo

\begin{tabular}{|l|c|c|c|c|}
\hline Muestra & Lab. No. & $\begin{array}{c}\text { Prof. } \\
(\mathrm{cm})\end{array}$ & ${ }^{14} \mathrm{C}(\mathrm{BP})$ & $\begin{array}{c}\text { cal. } \mathrm{AD} / \mathrm{BC}(2 \sigma) \\
95.4 \%\end{array}$ \\
\hline RAS-1 & Beta-219292 & $15-20$ & $50 \pm 40$ & $\begin{array}{c}1680 \mathrm{AD}(23.3 \%) 1730 \mathrm{AD} \\
1800 \mathrm{AD}(68.8 \%) 1930 \mathrm{AD} \\
1950 \mathrm{AD}(3.3 \%) 1960 \mathrm{AD}\end{array}$ \\
\hline RAS-2 & Beta-219293 & $30-33$ & $920 \pm 50$ & $1020 \mathrm{AD}(95.4 \%) 1220 \mathrm{AD}$ \\
\hline RAS-3 & GdA-844 & $58-61$ & $2455 \pm 35$ & $\begin{array}{l}760 \mathrm{BC}(24.6 \%) 680 \mathrm{BC} \\
670 \mathrm{BC}(70.8 \%) 410 \mathrm{BC}\end{array}$ \\
\hline
\end{tabular}

Tabla 1. Dataciones ${ }^{14} \mathrm{C}$.

Radiocarbon dating ${ }^{14} \mathrm{C}$. 
Pinus sylvestris el taxón más abundante. Se encuentran los mayores valores de Betula (5\%) aumentando progresivamente hacia techo. Dentro de los taxones higro-hidrófilos destaca Cyperaceae, que experimenta un aumento progresivo hacia el techo, alcanzando valores cercanos al $15 \%$. De los palinomorfos no polínicos predomina el tipo 170 y se detecta el aumento progresivo del tipo 181 .

ZONA II: abarca la parte media del diagrama polínico $(31-15 \mathrm{~cm})$, datada entre $920 \pm 50$ BP y $50 \pm 40$ BP. Desde el punto de vista litológico, de muro a techo, pasa de limos arenosos a arenas limosas. A lo largo de ella se produce un aumento en los componentes arbóreos y arbustivos representados en un incremento en Pinus sylvestris tipo y Erica arborea respectivamente. Hay un notable incremento de los taxones higro-hidrófilos específicamente Cyperaceae, que alcanza porcentajes cercanos al $40 \%$, siendo los más altos del perfil. Hacia el techo de la zona polínica se observa la disminución de algunos de los componentes herbáceos como Poaceae, Fabaceae y Plantago lanceolata. El componente arbóreo alcanza porcentajes del orden del $20 \%$ siendo Pinus sylvestris el taxon más abundante. Se encuentran también representados elementos de ribera (Alnus, Fraxinus y Corylus) y algunas presencias de Betula, Quercus ilex y Q. pyrenaica. En esta zona los valores de Pseudoschizaea circula son los más altos (30\%); además se observa un aumento en los tipos 140, 207, 51, 368 y 731.

ZONA III: se define para los $15 \mathrm{~cm}$ superiores y está caracterizada por la naturaleza orgánica de su sedimento; su formación se situa entre $50 \pm 40$ BP hasta la actualidad. Desde el punto de vista polínico destaca el incremento del componente arbóreo regional, constituido básicamente por Pinus sylvestris y $P$. pinaster y en menor proporción por Quercus ilex. Se observa una disminución importante del componente herbáceo, reflejada en el descenso de Cichorioideae, Asteraceae, Chenopodiaceae/Amaranthaceae y Plantago lanceolata acompañada por el aumento de $U r$ tica dioica en los últimos $10 \mathrm{~cm}$ del diagrama. Los taxones higro-hidrófilos específicamente Cyperaceae, vuelven a disminuir hasta alcanzar porcentajes inferiores al 5\%. Los tipos 55A, 113, 140, 207, 731 y Pseudoschizaea circula sobresalen por su abundancia en el diagrama.

Del sedimento se tomaron valores del $\mathrm{pH}$ comprendidos entre 5,4 y 6,2 y de la conductividad entre 10 y 160 $\mu \mathrm{S} / \mathrm{cm}$.

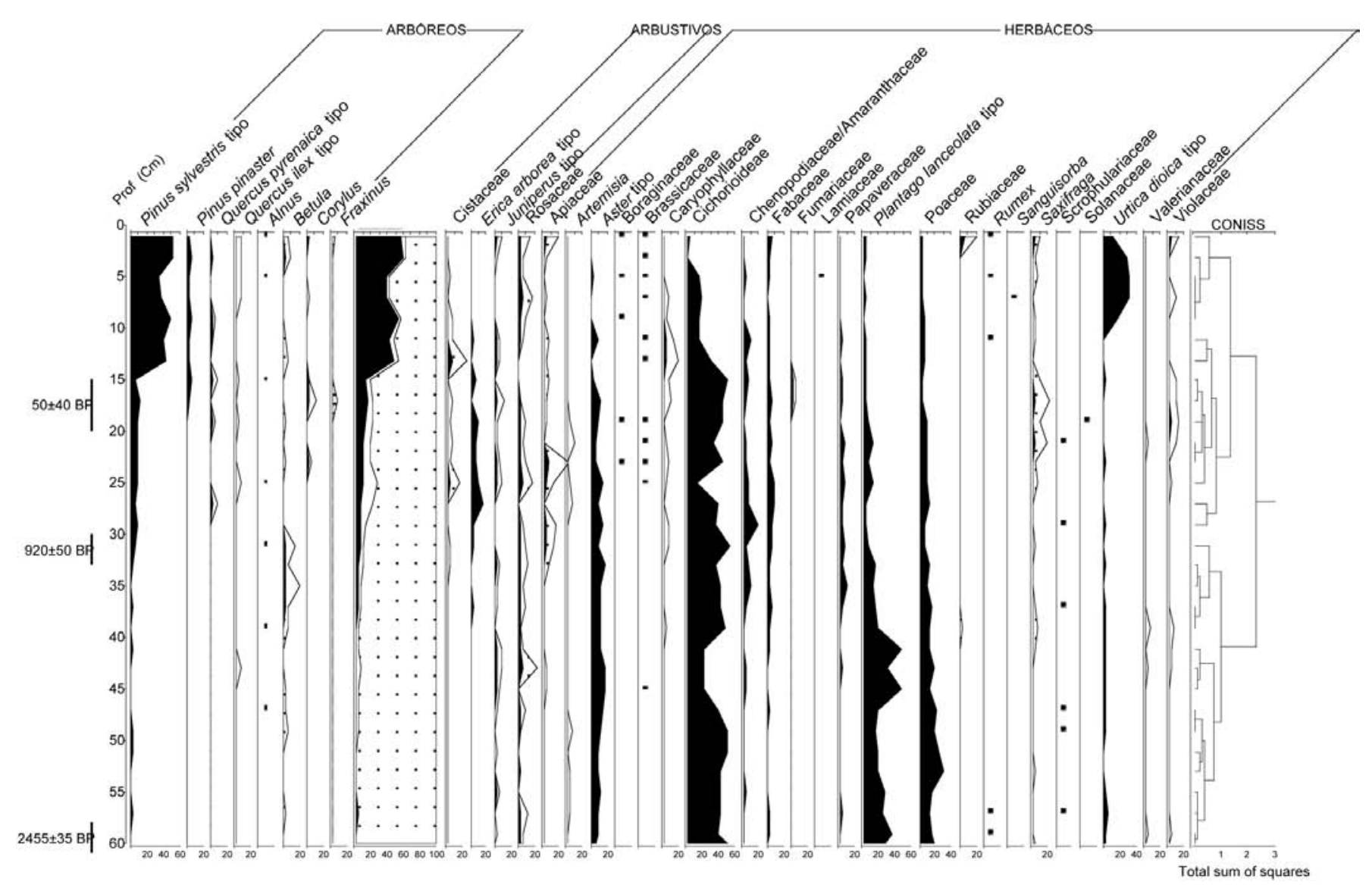

Figura 2. Diagrama polínico de la secuencia RAS (Taxones arbóreos/arbustivos/herbáceos). Pollen diagram of RAS sequence (arboreal/shrubs/herbs taxa). 


\section{INTERPRETACIÓN}

Las características de la dinámica vegetal observada en la secuencia RAS permiten determinar variaciones en las condiciones tróficas de la turbera. Este hecho queda manifestado en el diagrama por la presencia del tipo polínico 170 que permite sugerir unas condiciones meso-oligotróficas para el intervalo entre los $31 \mathrm{y} 60 \mathrm{~cm}$ de profundidad. Así mismo la asociación de los tipos 140, 181, $182,332 \mathrm{D}, 353 \mathrm{~A}$ y 731 sugiere unas condiciones mesoeutróficas para los últimos $31 \mathrm{~cm}$ de la turbera. Se puede observar el aumento del tipo 181 en la zona de cambio trófico de la turbera.

Este paso de condiciones oligotróficas (pobre en nutrientes) a meso-eutróficas (con abundancia de nutrientes) en torno a $920 \pm 50 \mathrm{BP}$ puede explicarse por el aumento en residuos naturales y/o antrópicos ricos en fosfatos y nitratos. Se observa en el diagrama polínico la coincidencia en el aumento de los tipos 140 y 781 con el tipo 351 indica- dor de ocupación humana. Además en la fase de cambio se observa la actividad humana a partir de la expansión de Poaceae, Fabaceae y el aumento de taxones nitrófilos antrópicos (Cichorioideae, Asteraceae, Papaveraceae, Boraginaceae y Rumex) y nitrofilos antropozoógenos (Plantago lanceolata, Urtica dioica, Chenopodiaceae/Amaranthaceae y Rubiaceae). Los tipos polínicos de afinidad coprófila (55A, 113 y 368) también refuerzan la interpretación.

Los datos de $\mathrm{pH}$ muestran el predominio de condiciones ácidas relacionadas con el tipo de suelo, proveniente de la degradación de las rocas del entorno (gneis). Además, que el aumento en la curva del pinar coincide con el incremento de acidez en el medio $(920 \pm 50$ BP y $50 \pm 40$ $\mathrm{BP})$, hecho que puede ser explicado por la acidificación del suelo producida por la acumulación de acículas de pino en el nivel más superficial del suelo. Además se evidencia la relación entre el cambio en las condiciones tróficas de la turbera, al pasar de oligotróficas a meso-eutróficas, y el aumento en la conductividad, probablemente relacionada

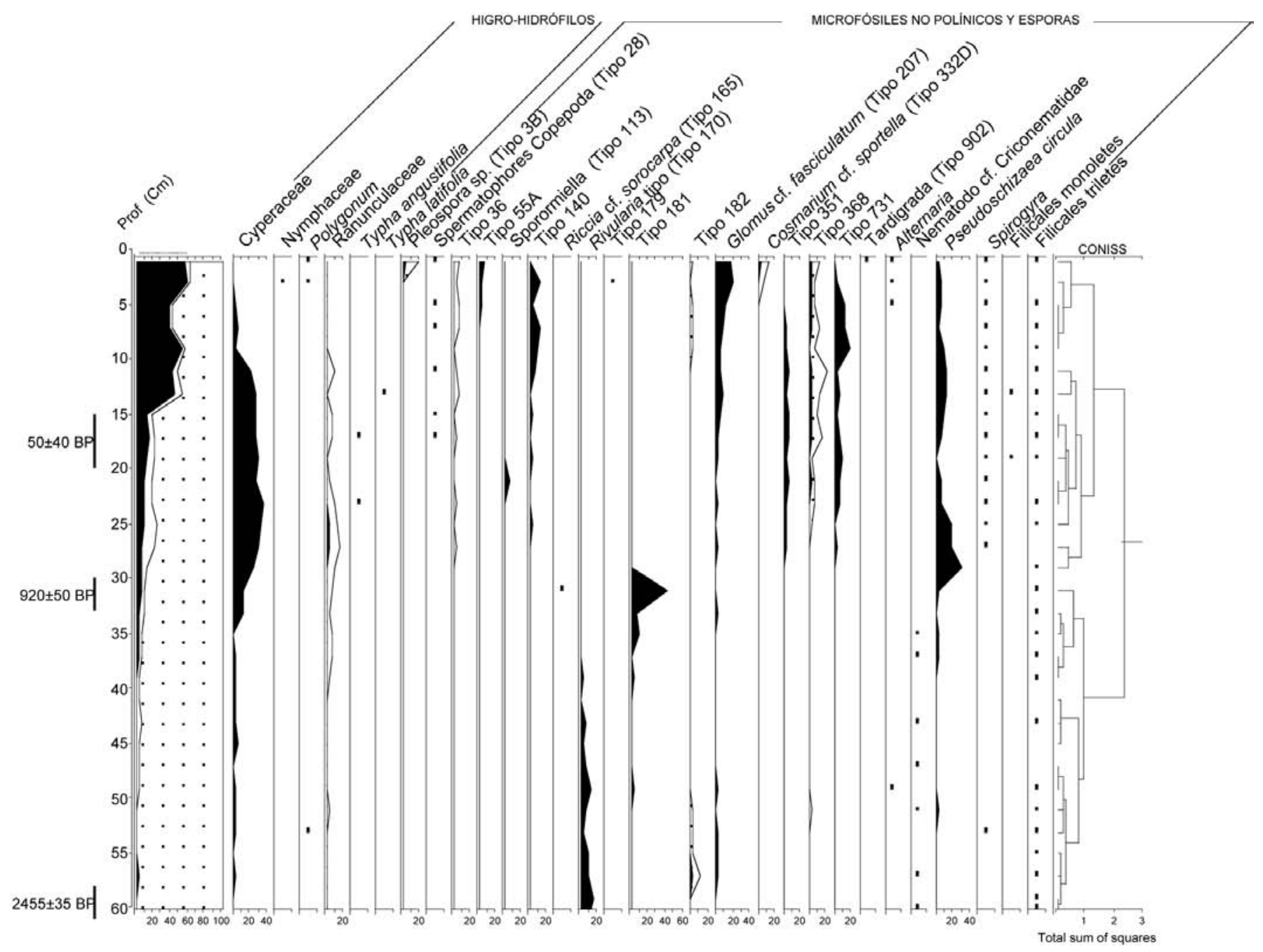

Figura 3. Palinomorfos de la secuencia RAS (Taxones higro-hidrófilos/Microfósiles no polínicos y esporas). Palynomorph diagram of RAS sequence (aquatic taxa/Non-pollen palynomorphs). 
con el incremento de iones disueltos en el medio favorecido por la presión ganadera.

El aumento de Artemisia y Chenopodiaceae coincide con el aumento de Cyperaceae; ya que la instalación de unas condiciones secas provoca la reducción del área encharcada, la cual es colonizada por Cyperaceae.

Las zonas de expansión de Plantago lanceolata siempre coinciden con un descenso en el porcentaje de Cichoriodeae; la presión pastoril, evidenciada en el aumento de Plantago lanceolata, genera una disminución en la cantidad nutrientes lo que se traduciría en el descenso de Cichorioideae, Poaceae y Papaveraceae.

En cuanto a la vegetación arbórea, se puede decir que Pinus sylvestris, dominante en el diagrama, es uno de los componentes regionales de la vegetación, probablemente su aumento progresivo $(50 \pm 40 \mathrm{BP})$ esté relacionado con las campañas de repoblación iniciadas a raíz de la implementación de la Ley de 1877, de siembras y plantaciones, que propició la extensión antrópica de los pinares en el área (Bauer, 1991; Gil, 1992).

Las Zona Polínica I (2455 \pm 50 BP-920 \pm 50 BP) corresponderían a una presión ganadera esporádica, posiblemente relacionada con la trashumancia estival. La mayor antropización del entorno quedaría reflejada en las Zonas polínicas II y III ya que aumenta la curva de Urtica dioica y de Rubiaceae que indican una presencia más constante del ganado en el medio. El incremento del pastoreo se refleja en la posible presencia del ganado a lo largo de todo el año, no únicamente en el verano y por tanto representa un aprovechamiento mayor del uso del suelo (Galop et al., 2003). Hechos similares se detectan en la Tolla de El

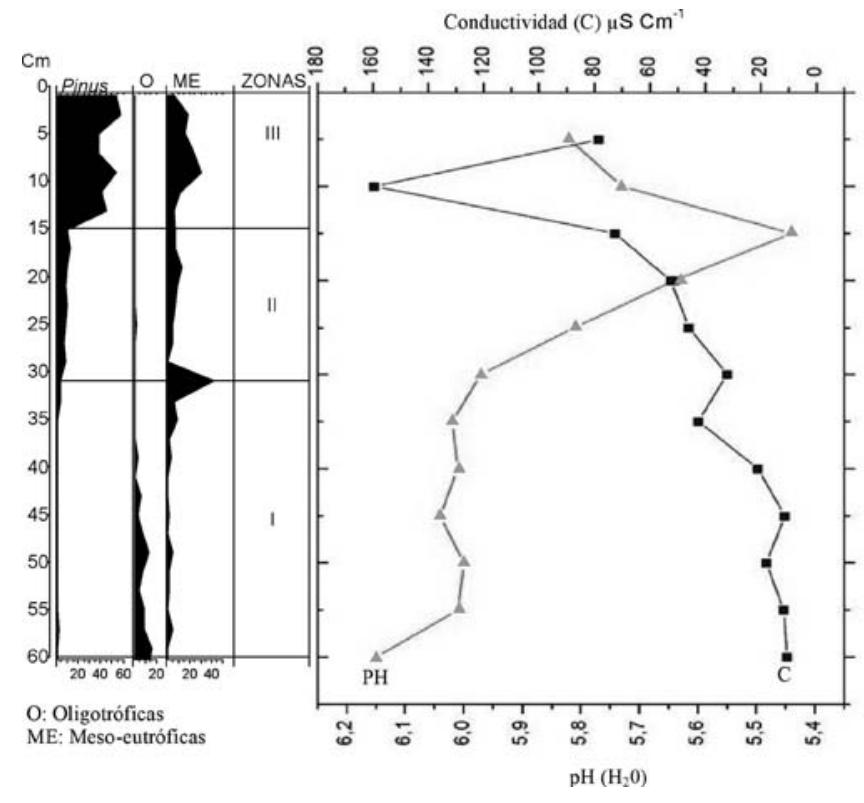

Figura 4. Relación Pinus-microfósiles no polínicos de afinidad oligotrófica y meso-eutrófica-pH-conductividad.

Relation between Pinus-oligotrophics and meso-eutrophics non-pollen palynomorphs-pH-conductivity.
Berrueco (Ruiz et al., 2006) situado a 25 kilómetros al oeste de la zona de estudio. El tipo 207, Glomus cf. fasciculatum, puede indicar fases erosivas posiblemente relacionadas al sobrepastoreo en la Zona III.

\section{CONCLUSIONES}

A la vista de todo lo expuesto podemos relacionar la dinámica vegetal del depósito higroturboso RAS con un clima mediterráneo en el que se detectan variaciones en la tasa de humedad y la disponibilidad de agua en el medio reflejadas en el aumento/disminución de los taxones xéricos e higro-hidrófilos.

Se concluye la presencia en el área de pastizales vivaces antropozoógenos dedicados a la explotación de ganado y que han sufrido una dinámica de cambio, representada en la presencia/ausencia, aumento/disminución de los taxones asociados a esta afinidad ecológica. Lo anterior se deduce del estudio realizado de los tipos polínicos: taxones nitrófilos antrópicos (Cichorioideae, Asteraceae, Papaveraceae, Boraginaceae y Rumex) y antropozoógenos (Plantago lanceolata, Urtica dioica, Chenopodiaceae/Amaranthaceae y Rubiaceae); y de los palinomorfos no polínicos: tipos $55 \mathrm{~A}, 113,165$ y 368.

Las fluctuaciones en los valores del $\mathrm{pH}$ y de la conductividad del sedimento se pueden explicar por las variaciones en las condiciones tróficas de la turbera y en los cambios detectados en la vegetación y en los usos del suelo.

\section{AGRADECIMIENTOS}

Nuestro más sincero agradecimiento al Parque Regional de Peñalara por las facilidades que nos han proporcionado en nuestras campañas de campo. A la familia Bartolomé por permitirnos el acceso a la finca de su propiedad donde se realizó el sondeo. A los revisores de este trabajo por sus aportaciones. Este trabajo se ha podido realizar gracias al proyecto de investigación CAM-UAH2005/041.

\section{REFERENCIAS}

Ahmed, S.E. \& Cain, R.F. 1972. Revision of the genera Sporormia and Sporormiella. Canadian Journal of Botany, 50, 419-477.

Almeida-Lenero, L., Hooghiemstra, H., Cleef, A.M. \& van Geel, B. 2005. Holocene climatic and environmental change from pollen records of lakes Zempola and Quila, central Mexican highlands. Review of Palaeobotany and Palynology, 136, 63-92.

Arobba, D. 1979. Determinazione di "Pinus halepensis" Miller e "Pinus pinaster" Aiton sulla base di differenze palinologiche. Archivio Botanico e Biogeografico Italiano, 55 (3), 83-92. 
Bakker, M. \& van Smeerdijk, D.G. 1982. A palaeoecological study of a Late Holocene section from "Het Ilperveld", Western Netherlands. Review of Palaeobotany and Palynology, 36, 95-163.

Bauer, 1991. Los montes de España en la historia. Servicio de Publicaciones Agrarias. Ministerio de Agricultura, Madrid, $610 \mathrm{pp}$.

Blackford, J.J. \& Innes, J.B. 2006. Linking current environments and processes to fungal spore assemblages: surface NPM data from woodland environments. Review of $\mathrm{Pa}$ laeobotany and Palynology, 141, 179-187.

Bronk-Ramsey, C. 1995. Radiocarbon Calibration and Analysis of Stratigraphy: The OxCal Program. Radiocarbon, 37, 425-430.

Bronk-Ramsey, C. 2001. Development of the Radiocarbon Program OxCal. Radiocarbon, 43 (2A), 355-363.

Cambón, G. 1981. Relations entre le contenu pollinique de l'atmosphere et le couvert vegetal mediterrannee occidentale a Montpellier (France), Valencia (Espagne) et Oran (algerie). Tesis Doctoral, Université des Sciences et Techniques du Languedoc, 105 pp. (inédita).

Carrión, J.S. 2001. Pastoreo y vulnerabilidad de la vegetación en la alta montaña mediterránea durante el Holoceno. Cuadernos de Geografía, 69/70, 7-22.

Cugny, C. 2004. L'apport des microfósiles non-polliniques à l'histoire de l'environnemet Pyrénéen. Entre référentiel actuel et reconstitution paléoenvironnementale. Une nouvelle approche appliquée à la reconstitution paléoenvironnementale du massif d'Iraty (Pays Basque). DEA, Université de Touluse-Le mirail, 186 pp. (Inédito).

Díaz-Laviada, T. 1989. Variabilidad del grano de polen en especies ibéricas del género Pinus. Tesis Doctoral, Universidad Politécnica de Madrid, 140 pp. (inédita).

Faegri, K., Kaland, P.E. \& Krzywinski, K.J. 1989. Textbook of Pollen Analysis. John Wiley and Sons, New York, $328 \mathrm{pp}$.

Fernández, F. 1988. Estudio florístico y fitosociológico del Valle del Paular (Madrid). Tesis Doctoral, Universidad Complutense de Madrid, 759 pp. (inédita).

Franco, F. 1995. Estudio palinológico de turberas holocenas en el Sistema Central: reconstrucción paisajística y acción antrópica. Tesis Doctoral, Universidad Autónoma de Madrid, 391 pp. (inédita).

Franco, F., García Antón, M. \& Sainz Ollero, H. 1998. Vegetation dynamics and human impact in the Sierra de Guadarrama, Central System, Spain. The Holocene, 8, 69-82.

Galop, D., Mazier, F., López Sáez, J.A. \& Vannière, B. 2003. Palynologie et histoire des activités humaines en milieu montagnard. Bilan provisoire des recherches et nouvelles orientations méthodologiques sur le versand nord des Pyrénées. Archéologie du Midi Médiéval, 21, 159-170.

Gil, M.J. 1992. Dinámica de la paleovegetación en el sector oriental del Sistema Central español durante el Holoceno, en base al análisis polínico. Implicaciones climáticas. Tesis Doctoral, Universidad de Alcalá de Henares, 211pp. (inédita).

Grimm, E.C. 1987. CONISS: a FORTRAN 77 program for stratigraphically constrained cluster analysis by the method of incremental sum of squares. Computers \& Geosciences, 13, 13-35.

Haas, J.N. 1996. Neorhabdocoela oocytes - palaeoecological indicators found in pollen preparations from Holocene freshwater lake sediments. Review of Palaeobotany and Palynology, 91, 371-382.

López Sáez, J.A. \& López García, P. 2003. Análisis palinológico del poblado calcolítico de Los Itueros (Santa María del Arroyo, Valle Amblés, Ávila, España). Trabalhos de Antropologia e Etnologia, 43, 171-180.

López Sáez, J.A., van Geel, B., Farbos-Texier, S. \& Diot, M.F. 2000. Aplicación de los microfósiles no polínicos en Palinología Arqueológica. In: Contributos das Ciências e das Technologias para a Arqueologia da Península Ibérica (Ed. J. Oliveira). Adecap, Porto, 11-20.

López Sáez, J.A., López Merino, L., Cugny, C. \& Galop, D. 2005. Paleoecología de medios húmedos: el aporte de los microfósiles no polínicos. Nuestra Tierra, 3, 13-15.

Marinova, E. \& Atanassova, J. 2006. Anthropogenic impact on vegetation and environment during the Bronze Age in the area of Lake Durankulak, NE Bulgaria: pollen, microscopic charcoal, non-pollen palynomorphs and plant macrofossils. Review of Palaeobotany and Palynology, 141, 165-178.

Mateus, J.E. 1992. Holocene and present-day ecosystems of the Carvalhal Region, Southwest Portugal. Tesis doctoral. Universidade de Utreque, 184 pp. (inédita).

Moore, P.D., Webb, J.A. \& Collison, M.E. 1991. Pollen analysis. Blackwell Scientific Publications, Oxford, 216 pp.

Pals, J.P., van Geel, B. \& Delfos, A. 1980. Palaeoecological studies in the Klokkeweel bog near Hoogkarspel (Prov. of Noord-Holland). Review of Palaeobotany and Palynology, 30, 371-418.

Peinado Lorca, M. \& Rivas-Martínez, S. 1987. La Vegetación de España. Servicio de Publicaciones de la Universidad de Alcalá, Alcalá de Henares, 544 pp.

Prager, A., Barthelmes, A., Theuerkauf, M. \& Joosten, H. 2006. Non-pollen palynomorphs from modern Alder carrs and their potential for interpreting microfossil data from peat. Review of Palaeobotany and Palynology, 141, 7-31.

Reille, M. 1992. Pollen et Spores d'Europe et d'Afrique du Nord. Laboratoire de botanique historique et palynologie, Marseille, $520 \mathrm{pp}$.

Riera, S., Lopez-Saez, J.A \& Julia, R. 2006. Lake responses to historical land use changes in northern Spain: the contribution of non-pollen palynomorphs in a multiproxy study. Review of Palaeobotany and Palynology, 141, 127-137.

Ruiz, B., Gómez, C., López, J.A., Dorado, M., Valdeolmillos, A., \& Gil, M.J. 2006. Paleoambiente y usos del suelo durante el Holoceno reciente en la Tolla Collado de el Berrueco (Sierra de Guadarrama Madrid). Geogaceta, 40, 227-230.

Uzquiano, P. 2002. Vegetation and firewood management at Cueva de la Vaquera (Segovia, Spain) between 6 and 3.7 Kyr. BP: anthracological contribution to the landscape archaeology of the Spanish Central Mountains. In: Charcoal 
análisis methodological approaches, palaeoecological results and Word uses (Ed. S. Thiébault). Bar internacional series, Paris, 109-112.

Valdés, B., Díez, M.J. \& Fernández, I. 1987. Atlas Polínico de Andalucía Occidental. Instituto de Desarrollo Regional de la Universidad de Sevilla, Excma. Diputación de Cádiz, Cádiz, 450 pp.

van der Wiel, A.M. 1983. A palaeoecological study of a section from the foor of the hazendonk (Zuid-Holland, The Netherlands), based on the analysis pollen, spores and macroscopic plant remains. Review of Palaeobotany and Palynology, 38, 35-90.

van Geel, B. 1978. A palaeoecological study of Holocene peat bog sections in Germany and The Netherlands. Review of Palaeobotany and Palynology, 25, 1-120.

van Geel, B. 1986. Application of fungal and algal remains and other microfossils in palynological analyses. In: Handbook of Holocene Palaeoecology and Palaeohydrology (Ed. B.E. Berglund). John Wiley \& Sons Ltd., Chichester, 497-505.

van Geel, B. 2001. Non-pollen palynomorphs. In: Tracking environmental change using lake sediments; volume 3: Terrestrial, algal and silicaceous indicators (Eds. J.P. Smol, H.J.B. Birks \& W.M. Last). Kluwer Academic Publishers, Dordrecht, 99-119.
Van Geel, B. \& Aptroot, A. 2006. Fossil ascomycetes in Quaternary deposits. Nova Hedwigia, 82, 313-329.

van Geel, B., Bohncke, S.J.P. \& Dee, H. 1981. A palaeoecological study of an Upper Late Glacial and Holocene sequence from 'De Borchert', The Netherlands. Review of Palaeobotany and Palynology, 31, 367-448.

van Geel, B., Hallewas, D.P. \& Pals, J.P. 1983. A Late Holocene deposit under the Westfriese Zeedijk near Enkhuizen (Prov. of Noord-Holland, The Netherlands): Palaeoecological and archaeological aspects. Review of Palaeobotany and Palynology, 38, 269-335.

van Geel, B., Coope, G.R. \& van der Hammen, T. 1989. Palaeoecology and stratigraphy of the Lateglacial type section at Usselo (The Netherlands). Review of Palaeobotany and Palynology, 60, 25-129.

van Geel, B., Buurman, J., Brinkkemper, O., Schelvis, J., Aptroop, A., van Reenen, G. \& Hakbijl, T. 2003. Environmental reconstruction of a Roman Period settlement site in Uitgeest (The Netherlands), with special reference to coprophilous fungi. Journal of Archaeological Science, 30, 873-883.

Manuscrito recibido: 15 de noviembre, 2006 Manuscrito aceptado: 17 de abril, 2007 